\title{
Effect of spin-orbital interaction on continuous and jumpwise reversal magnetization in $\left[\mathrm{Mn}(\mathrm{II})(\mathrm{HL})\left(\mathrm{H}_{2} \mathrm{O}\right)\right]\left[\mathrm{Mn}(\mathrm{III})(\mathrm{CN})_{6}\right] \cdot 2 \mathrm{H}_{2} \mathrm{O}$ molecular ferrimagnet
}

\author{
Marina Kirman ${ }^{*}$ and Roman Morgunov \\ Institute of Problems of Chemical Physics of Russian Academy of Sciences, 142432, Chernogolovka, Russia
}

\begin{abstract}
Magnetic jumps were revealed during the magnetization reversal of $\left[\mathrm{Mn}(\mathrm{II})(\mathrm{HL})\left(\mathrm{H}_{2} \mathrm{O}\right)\right]$ $\left[\mathrm{Mn}(\mathrm{III})(\mathrm{CN})_{6}\right] \cdot 2 \mathrm{H}_{2} \mathrm{O}$ molecular ferrimagnet. Amplitudes of the jumps were $0.01-0.1 \%$ of the saturation magnetization. Fourier transform of the time series of jumps magnetization indicates that frequency spectrum is close to the white noise. Statistic distribution of the jumps versus time reveals the appearance of the most jumps at the beginning of the demagnetization. Effect of spin-orbit coupling on statistical distribution of magnetization jumps was considered by comparison of two compounds with different single ion anisotropies. The increase of spin-orbit interaction leads to the decrease of power of spectral density of magnetization jumps.
\end{abstract}

\section{Introduction}

One of the most interesting new classes of materials is molecular magnets. The exchange interaction in molecular magnets occurs between the metal atoms through organic $\mathrm{C}-\mathrm{O}, \mathrm{C}-\mathrm{N}$ bridging and other organic ligands. The atomic structures of these chains and their lengths, as well as the chemical compositions, are variable that attracts researchers of molecular magnet chemistry. However, the interest of physicists is searching of new magnetic effects in these artificial materials. In addition, the ratio between their magnetic characteristic parameters (the domain wall width, the exchange radius, etc.), and geometry of the crystal lattice (lattice parameters) are different from the conventional inorganic magnets. Mentioned above feature of the metal organic magnets lead to the unusual effects, such as a postponed emission of magnetic noise detected in $\mathrm{K}_{0.4}\left[\mathrm{Cr}(\mathrm{CN})_{6}\right][\mathrm{Mn}(\mathrm{R} / \mathrm{S})-\mathrm{pn}](\mathrm{R} / \mathrm{S})-\mathrm{pnH}_{0.6} \quad$ chiral ferrimagnet (Yellow needle, (YN)) $[1,2]$. The novelty of this phenomenon is that these magnetization jumps occur in a constant magnetic field, while Barkhausen jumps occur in a sweeping magnetic field. This fact allows us to consider magnetization jumps as spontaneous nonequilibrium events manifesting non-linear effects in the motion of the domain wall or other spin ensembles (spin soliton and skyrmions in YN were discussed in [3, 4]). The physical nature of jumps in the magnetic reversal constant field is not clear.

In this paper we were focused on searching of similar phenomena (stepwise demagnetization) in other types of molecular magnets, which structure is similar to early studied YN, but driving parameters (exchange interaction, spin-orbital coupling etc) can be varied. Chemical design allows one to create a series of such analogues possessing similar crystal structure and magnetic properties. Thus, metal atoms in studied compound were replaced by other atoms as well as exchange bridges were restructured. The main difference of $\quad\left[\mathrm{Mn}(\mathrm{II})(\mathrm{HL})\left(\mathrm{H}_{2} \mathrm{O}\right)\right]\left[\mathrm{Mn}(\mathrm{III})(\mathrm{CN})_{6}\right] \cdot 2 \mathrm{H}_{2} \mathrm{O} \quad$ (Brown Needle - BN) compound studied in this paper with those studied in $[1,2]$ is strong spin-orbit interaction in $\mathrm{Mn}^{3+}$ ions (single-ion anisotropy $D \sim 10 \mathrm{~cm}^{-1}$ [5]). In the previously studied YN magnets, weak spin-orbit coupling of $\mathrm{Mn}^{2+}$ and $\mathrm{Cr}^{3+}$ ions took place (single-ion anisotropy of $\mathrm{Mn}^{2+}$ is $D \sim 0.004 \mathrm{~cm}^{-1}$ [6]). Thus, the aim of our work is to establish the laws of the continuous and jumpwise parts of the relaxation in the magnetization reversal $\left[\mathrm{Mn}(\mathrm{II})(\mathrm{HL})\left(\mathrm{H}_{2} \mathrm{O}\right)\right] \quad\left[\mathrm{Mn}(\mathrm{III})(\mathrm{CN})_{6}\right] \cdot 2 \mathrm{H}_{2} \mathrm{O}$ molecular magnets.

\section{Experiment}

Method of synthesizing [Mn(II)(HL) $\left.\left(\mathrm{H}_{2} \mathrm{O}\right)\right]$ $\left[\mathrm{Mn}(\mathrm{III})(\mathrm{CN})_{6}\right] \cdot 2 \mathrm{H}_{2} \mathrm{O}(\mathrm{BN})$ microcrystals is described in detail in [7]. The compound (C9H17N8O3Mn2) with the formula weight $=395.16 \mathrm{~g} / \mathrm{mol}$ was synthesized as darkred plates $(0.28 \times 0.12 \times 0.05 \mathrm{~mm})$, orthorhombic, $\mathrm{a}=$ 7.5145(5) $\AA, \mathrm{b}=14.261(1) \AA, \mathrm{c}=14.8844(1) \AA, \mathrm{V}=$ 1590.7(2) $\AA^{3}, Z=4$.

The crystal structure of $\mathrm{BN}$ is quasi-two-dimensional layers of alternating $\mathrm{Mn}^{2+}$ ions and $\mathrm{Mn}^{3+}$ linked by $-\mathrm{CN}$ -

Corresponding author: kirmanm@yandex.ru 
cyanide bridges (Fig. 1). The individual layers are connected by van der Waals forces. The chiral ligand $\mathrm{L}$ = R-pn (1,2-diaminopropane) is in the interlayer space. Space group of $\mathrm{BN}$ crystals is chiral $\left(P 2_{1} 2_{1} 2_{1}\right)$. Curie temperature is $T_{\mathrm{C}}=21.2 \mathrm{~K}$, saturation magnetization is $M_{\mathrm{S}}=2.79 \mathrm{emu} / \mathrm{g}$ at $T=20 \mathrm{~K}, M_{\mathrm{S}}=3.94 \mathrm{emu} / \mathrm{g}$ at $T=2$ $\mathrm{K}$. The coercive force is $H_{\mathrm{C}}=400 \mathrm{Oe}$ at $T=2 \mathrm{~K}$.

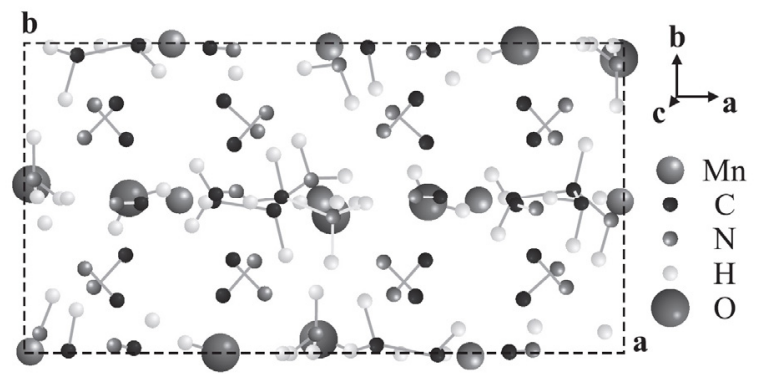

Fig. 1. Atomic structure of $\left[\mathrm{Mn}(\mathrm{II})(\mathrm{HL})\left(\mathrm{H}_{2} \mathrm{O}\right)\right]\left[\mathrm{Mn}(\mathrm{III})(\mathrm{CN})_{6}\right]$ molecular ferrimagnet [7].

Dependences of magnetic moment on time, field and temperature were recorded by a MPMS 5XL Quantum Design SQUID magnetometer. Measurements were performed in the magnetic field range from $+50 \mathrm{kOe}$ to $50 \mathrm{kOe}$ at temperature $T=2-300 \mathrm{~K}$. The temperature was stabilized with an accuracy of $0.1 \mathrm{~K}$ during the measurement of the sample. The experimental method of studying the dynamics of magnetization reversal is schematically shown in Fig. 2.

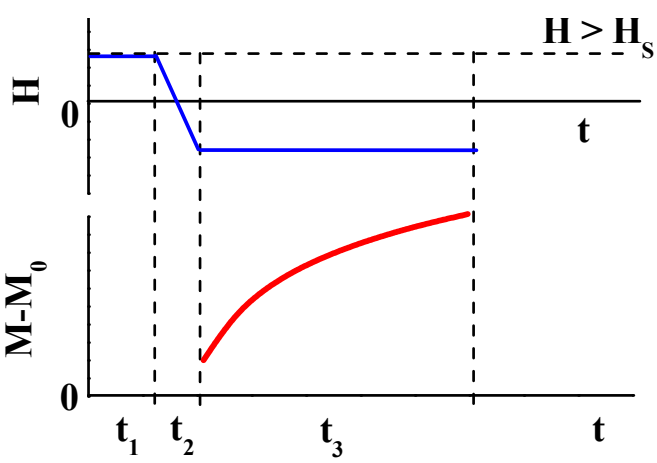

Fig. 2. The scheme of switching of the magnetic field $H$ (upper panel) and the corresponding response of the magnetization of sample $M$ (bottom panel).

Before measurements the sample was cooled down in zero magnetic field. Then the sample is magnetized during $t_{1}=300 \mathrm{~s}$ in saturation field $H=5 \mathrm{kOe}$ much larger saturation field of sample $H_{\mathrm{S}} \sim 1 \mathrm{kOe}$. After saturation the magnetic field of magnetometer was switched on against the vector of initial magnetization of the sample. "Dead time" necessary for field switching was $t_{2} \approx 50 \mathrm{~s}$. After magnetic field stabilization in "no overshoot" mode, the time dependence of the magnetic moment was measured during time $t_{3}$. On the bottom panel of Fig. 2 shows a time dependence of the magnetic moment $\left(M-M_{0}\right)$, where $M_{0}$ - the magnetic moment at the beginning of the measurement $M(t)$.

\section{Experimental results and discussion}

\subsection{Continuous component of magnetic relaxation}

Fig. 3 shows the time dependence of the magnetic moment $\left(M-M_{0}\right)(t)$. The magnetic field is switched in accordance with the scheme (Fig. 2) and directed against the vector magnetization of the sample. Switch on the field results in the appearance of the magnetic aftereffect, i.e. the change in the magnetization of the sample with time. If the relaxation of the magnetization associates with a single-barrier process, the magnetic moment is exponentially dependent on time. If there are barriers of different height, the time dependence of magnetization is logarithmic [8].

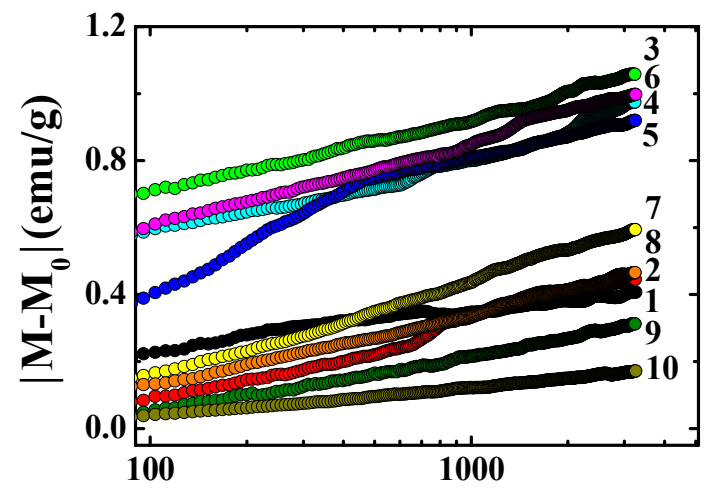

Fig. 3. Time dependences of the absolute value of the change in the magnetic moment $\left|M-M_{0}\right|$ at $T=10 \mathrm{~K}$ in different magnetic fields: +10 Oe (1), 0 Oe (2), -5 Oe (3), -30 Oe (4), -35 Oe (5), -40 Oe (6), -100 Oe (7), -200 Oe (8), -400 Oe (9), -1000 Oe (10).

Dependencies $M(t)$ shown on Fig. 3 were approximated by the formula:

$$
M=M_{0}-S \cdot \ln (t),
$$

where $S$ is magnetic viscosity. The resulting values of $S$ are calculated into a normalized magnetic viscosity $S_{\mathrm{V}}=$ $S / \chi_{\text {irr }}$ (Fig. 4), where $\chi_{\text {irr }}$ - irreversible magnetic susceptibility of the sample [9].

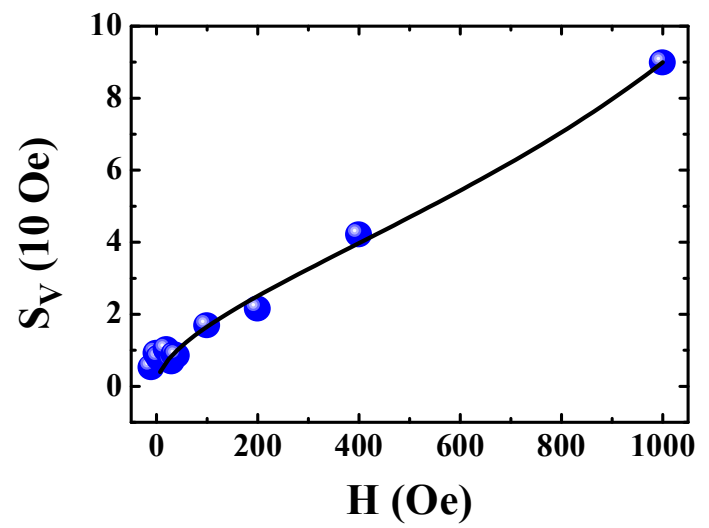

Fig. 4. Dependence of the normalized magnetic viscosity $S_{\mathrm{V}}$ on the magnetic field $H$ at a temperature $T=10 \mathrm{~K}$. The solid line shows the approximation by the expression (3). 
The value of $S$ depends strongly on the field, dependence of $S(H)$ has a maximum near the coercive force of the sample $H_{\mathrm{C}}=40 \mathrm{Oe}$ at $T=10 \mathrm{~K}$, which can be attributed to strong pinning of the domain wall by casual obstacles (structural defects) [10].

In the strong pinning limit, the activation energy of the relaxation given by equation [11]:

$$
E=(4 / 3) f b\left[1-\left(H / H_{0}\right)^{0.5}\right]^{1.5},
$$

where $f$ is the force required for a single depinning, $4 b$ is the thickness of the domain wall, $H_{0}$ is the threshold value of the magnetic field necessary for separation of the domain wall from a defect in the absence of thermal activation.

Dependence $S_{\mathrm{V}}(H)$ (Fig. 4) was approximated by the expression for the magnetic viscosity [12]:

$$
S_{V}=\left(k_{B} T / f b\right)\left(H H_{0}\right)^{0.5} /\left[1-\left(H / H_{0}\right)^{0.5}\right]^{0.5}
$$

The value $f b=4 \cdot 10^{-18} \mathrm{~J}$ and the value of the threshold magnetic field $H_{0}=1823 \pm 312$ Oe were determined from the approximation of the dependence $S_{\mathrm{V}}(H)$. The value $f b$ is corresponding to the work hardening of a single depinning of the domain wall from the obstacle. The value of the activation energy $E=5.33$ $\cdot 10^{-18} \mathrm{~J}$ was found in the case of strong pinning by the equation (2).

\subsection{Magnetization reversal jumps and statistical analysis}

The dependence $M(t)$ (Fig. 5) manifests the stochastic magnetization jumps. Statistical time distribution of the jumps probability $P$ and distribution of jump amplitudes $\Delta M$ are shown in the inset on Fig. 5 and on Fig. 6, respectively. The initial magnetic moment and the amplitude of the jumps were normalized on saturation magnetization $M_{\mathrm{s}}$.

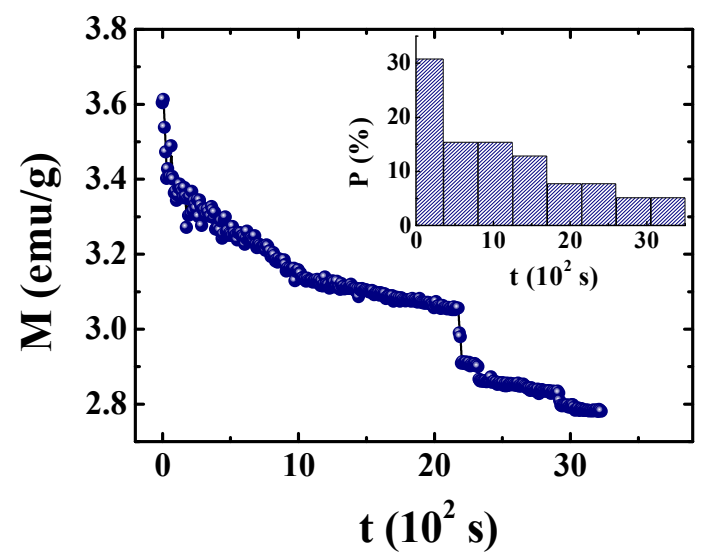

Fig. 5. The time dependence of the magnetic moment $M$ and the jumps probability $P$ (inset) at $H=-50 \mathrm{Oe}, T=10 \mathrm{~K}$.

The value of the correlation coefficient $r_{\mathrm{t} \Delta \mathrm{M}}=0.23$ between the time and the jump amplitude $\Delta M$ was found by the formula:

$$
r_{\mathrm{t} \Delta \mathrm{M}}=\operatorname{cov}(t, \Delta M) /\left(\delta_{\mathrm{t}} \delta_{\Delta M}\right),
$$

$\operatorname{cov}(t, \Delta M)$ is the covariance, $\sigma_{\mathrm{t}}$ и $\sigma_{\Delta \mathrm{M}}$ are the standard deviations of the $t$ and $\Delta M$, respectively. The correlation coefficient $r_{\mathrm{t} \Delta \mathrm{M}}$ value is found between 0 and 3 accordingly with Student's scale, which indicates absence of correlation between the time and the amplitude of the jump.

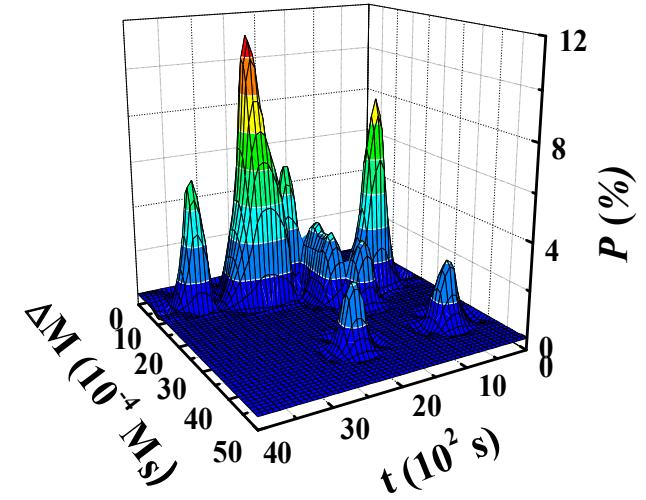

Fig. 6. The distribution of the amplitude $\Delta M$ and the time of jump appearance $t$ at $H=-50 \mathrm{Oe}, T=10 \mathrm{~K}$.

The distribution of the jumps amplitude $\Delta M$ (Fig. 6) show that most jumps occur at the beginning of the measurement.

The atomic structure of the samples is chiral due to Dzyaloshinsky-Moriya interaction. Noncollinear spin states can be formed in chiral molecular magnets. The magnetization of these states is smaller than the magnetization of the commensurate magnetically ordered state. In addition, the energies of noncollinear spin states are characterized by a discrete spectrum. Thus, the transition from a magnetically ordered to a magnetochiral state can be explained by the observed demagnetization jumps.

\subsection{Fourier analysis of demagnetization jumps}

The time sequence of jump amplitudes $\left(t_{\mathrm{n}}, \Delta M_{\mathrm{n}}\right)$ is shown in Fig. 7, where $n=1,2, \ldots, N$ is the number of the time interval $t_{\mathrm{n}}=n \cdot \Delta t, \Delta M_{\mathrm{n}}$ is the jump amplitude occurring in the interval $\left(t_{\mathrm{n}}-\Delta t / 2 ; t_{\mathrm{n}}+\Delta t / 2\right)$. Correlogram $r(k)$ of the time series $\left(t_{\mathrm{n}}, \Delta M_{\mathrm{n}}\right)$ was obtained by the described technique in [1].

The spectral density of the jumps $S(f)$ (inset on Fig. 7) was obtained by Fourier transform of the correlogram $r(k)$ in accordance with the Wiener-Khinchin theorem [13]:

$S\left(f_{n}\right)=\sum_{k=1}^{N} r(k) \cdot \exp \left(-(2 \pi i / N) k f_{n} \Delta t\right)$

where $f_{\mathrm{n}}=n /(N \Delta t)$ corresponds to the set of frequencies of the discrete Fourier transform. Frequency dependence of the spectral density $S(f)$ corresponds to white noise, which is typical for random processes $[14,15]$. 


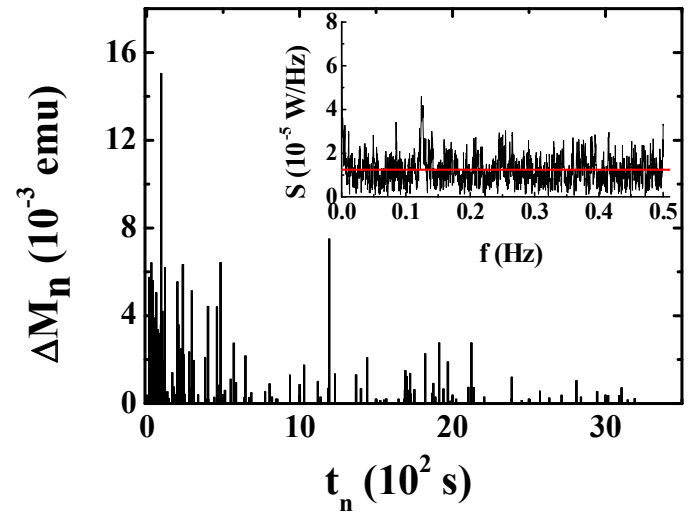

Fig. 7. The time sequence of jump amplitudes collected from 14 independent measurements of $M(t)$ at $H=-100 \mathrm{Oe}, T=10$ K. Inset: the spectral density $S$ of magnetization jumps in stationary magnetic field at $10 \mathrm{~K}$. The solid line shows the approximation by $(1 / f)^{\alpha}$ function, where $\alpha=0 \pm 0.1$.

White noise was detected as well in YN chiral ferrimagnet in stationary reverse magnetic field [1]. However spectral density of the stochastic magnetization jumps in $\mathrm{YN}$ was higher than spectral density in $\mathrm{BN}$ at similar conditions. Reduction of amplitude of magnetization jumps can be due to increase of spin-orbit interaction.

\section{Conclusions}

There are obtained data on the continuous and jumpwise reversal magnetization of $\left[\mathrm{Mn}(\mathrm{II})(\mathrm{HL})\left(\mathrm{H}_{2} \mathrm{O}\right)\right]$ $\left[\mathrm{Mn}(\mathrm{III})(\mathrm{CN})_{6}\right] \cdot 2 \mathrm{H}_{2} \mathrm{O}$ molecular magnet. The values of the magnetic viscosity as well as the activation energy corresponding to the domain wall switching regime were determined for a continuous demagnetization component. It was establish that the frequency spectrum of the jumps corresponds to white noise. The reversal of the magnetization reversal can be caused by the transition of the sample to a state with a magnetochiral structure as a result of competition between the Heisenberg symmetric exchange interaction and the Dzyaloshinsky-Moriya antisymmetric interaction. Spinorbit interaction effects power of spectral density of magnetization jumps and the energy barriers of domain movements.

This work was supported by RFBR No. 15-02-05149 a.

\section{References}

1. R.B. Morgunov, A.D. Talantsev, Phys. Rev. B 94, 144421 (2016)

2. A. Talantsev, M. Kirman, R. Morgunov, Phys. Status Solidi B 253, 1222 (2016)

3. R.B. Morgunov, M.V. Kirman, K. Inoue, Y. Tanimoto, J.I. Kishine, A.S. Ovchinnikov, and O. Kazakova, Phys. Rev. B 77, 184419 (2008)

4. M.V. Kirman, A.D. Talantsev, O.V. Koplak, and R.B. Morgunov, JETP Lett. 101, 398 (2015)
5. L.J. Challis, A.A. Chazi, K.J. Maxwell, J. Phys. C: Solid State Phys. 12, 303 (1979)

6. A. Edgar, E. Siegel and W. Urban, J. Phys. C: Solid St. Phys. 13, 6649 (1980)

7. W. Kaneko, S. Kitagawa, M. Ohba, JACS 129, 248 (2007)

8. D.K. Lottis, E.D. Dahlberg, J.A. Christner, J.I. Lee, R.L. Peterson, and R.M. White, J. Appl. Phys. 63, 2920 (1988)

9. D. Givord, Q. Lu, M.F. Rossignol, P. Tenaud, T. Viadieu, JMMM 83, 183 (1990)

10. P. Gaunt, J. Appl. Phys. 59, 4129 (1986)

11. P. Gaunt, Philos. Mag. B 48, 261 (1983)

12. A.I. Dmitriev, V.V. Kucheryaev, E.I. Kunitsyna, R.A. Valeev, R.B. Morgunov, V.P. Piskorskii, O.G. Ospennikova, E.N. Kablov, Physics of the Solid State 58, 1582 (2016)

13. G.M. Jenkins, D.G. Watts, Spectral analysis and its applications (Holden-Day, San Francisco, 1968)

14. H.D. I. Abarbane, R. Brown, J.J. Sidorowich, and L.Sh. Tsimring, Rev. Mod. Phys. 65, 1331 (1993)

15. N.J. Kasdin, Proceedings of the IEEE 83, 802 (1995) 